\title{
Complete thoracoscopic excision of an infected bronchogenic cyst due to mediastinitis
}

\author{
Yong Hwan Kim, Jae Jun Kim, Si Young Choi, Seong Cheol Jeong, In Sub Kim \\ Department of Thoracic and Cardiovascular Surgery, Uijeongbu St. Mary's Hospital, The Catholic University of Korea College of Medicine, \\ Uijeongbu, Gyeonggi-do, Korea \\ Correspondence to: Jae Jun Kim, MD, PhD. Department of Thoracic and Cardiovascular Surgery, Uijeongbu St. Mary's Hospital, Geumo-dong, \\ Uijeongbu, Gyeonggi-do 480-717, Korea. Email: medkjj@hanmail.net.
}

\begin{abstract}
Because bronchogenic cysts can result in infection, further complications due to compression of adjacent organs or malignant transformation, symptomatic and complicated bronchogenic cysts are usually surgically removed. However, surgical excision can be hazardous or incomplete because of dense adhesion to surrounding vital organs as a consequence of severe inflammation. Herein, we report a rare case of infected bronchogenic cyst caused by mediastinitis and we describe how a surgical approach using video-assisted thoracoscopic surgery (VATS) should be considered for treatment, even among patients with complicated bronchogenic cysts.
\end{abstract}

Keywords: Bronchogenic cyst; video-assisted thoracoscopic surgery (VATS); mediastinitis

Submitted Dec 01, 2016. Accepted for publication Apr 20, 2017.

doi: $10.21037 /$ jtd.2017.08.172

View this article at: http://dx.doi.org/10.21037/jtd.2017.08.172

\section{Case presentation}

A 41-year-old man was referred to our department for acute mediastinitis and a mediastinal infected cystic lesion. $\mathrm{He}$ was a habitual heavy drinker, had vomited four days prior, and fasted for four days. He presented with an aggravated sore throat and neck pain, and a fever. In addition, his neck (anterior low part) was reddish and swollen after vomiting. These were not responding to conservative treatment that included antibiotics. Chest radiographs showed a widened mediastinum without pneumonia, and chest computed tomography (CT) revealed cellulitis with fluid collection in the right anterior neck and a rim-enhancing cystic lesion $(6.2 \mathrm{~cm} \times 3.8 \mathrm{~cm} \times 2.8 \mathrm{~cm})$ in the anterosuperior mediastinum with perilesional infiltration, which caused a leftward deviation of the trachea, suggesting the patient had an infected mediastinal cyst accompanying acute mediastinitis and deep neck infection (Figure 1A,B). The patient's esophagogram showed no abnormal findings. Because his condition deteriorated rapidly and was refractory to conservative treatment, emergency drainage or a surgical approach were determined to be the best courses of action.
Because percutaneous drainage was clinically infeasible, we decided to take an immediate surgical approach using conventional 3-port video-assisted thoracoscopic surgery (VATS) with carbon dioxide insufflation. We found that the cyst had adhered to adjacent vital organs, especially the superior vena cava and the innominate vein. Because the cyst extended from the thymus, we determined that it was an infected thymic cyst. The cyst was well encapsulated and contained whitish pus (Figure $2 A$ ). We made a small hole in the cyst and suctioned out the contents. Suctioning the cyst reduced its size and, fortunately, secured a surgical plane, allowing us to completely remove the thymus with the shrunken cyst without difficulty (Figure 2B). Histopathologic examination revealed that the cyst wall was lined with ciliated pseudostratified columnar epithelia with inflammatory cell infiltration and it also consisted of fibrous tissue and acute inflammatory and hemorrhagic cells, suggesting an infected bronchogenic cyst (1). No bacterial growth was found in the fluid of the cyst. His condition was improved dramatically after surgery, and postoperative course was uneventful. He was discharged on the ninth postoperative day without any complications. Chest CT 

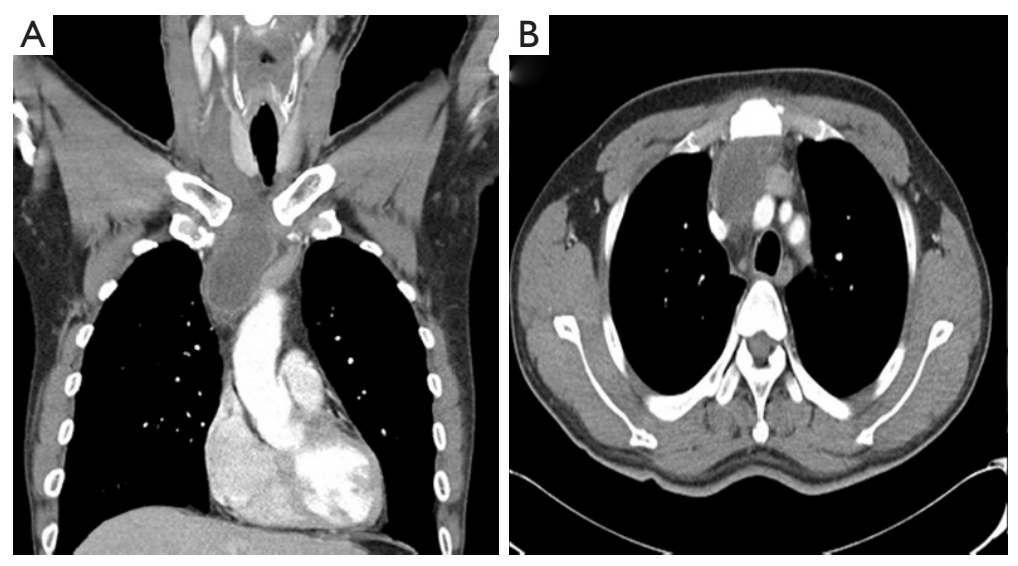

Figure 1 Preoperative chest CT (A,B) revealed cellulitis with fluid collection in the right anterior neck and a rim-enhancing cystic lesion $(6.2 \mathrm{~cm} \times 3.8 \mathrm{~cm} \times 2.8 \mathrm{~cm})$ in the anterosuperior mediastinum with peri-lesional infiltration deviating the trachea leftward, suggesting an infected mediastinal cyst accompanying acute mediastinitis and deep neck infection.
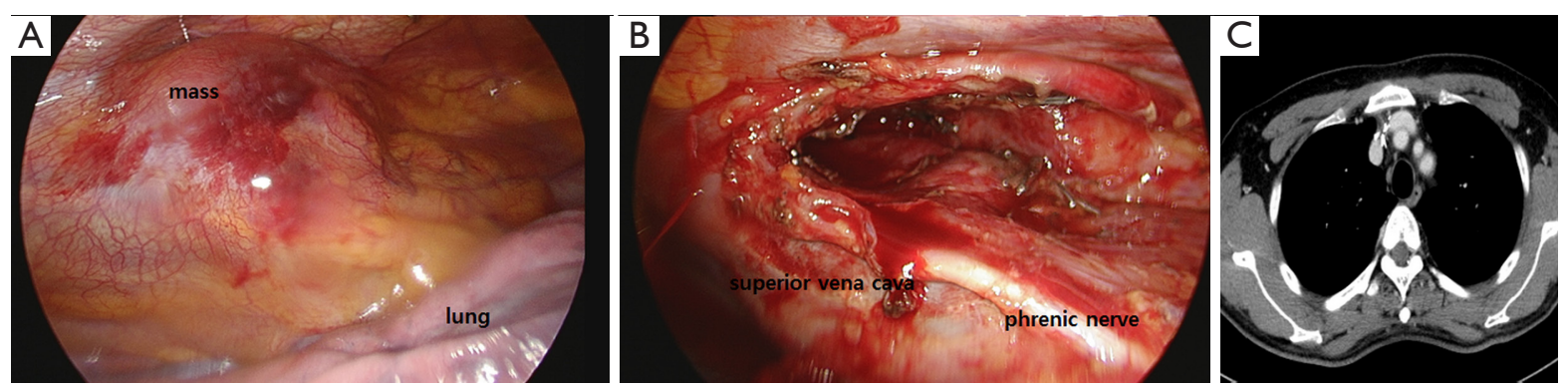

Figure 2 The cyst was well encapsulated and contained whitish pus (A). The thymus and the cystic mass were completely excised using video-assisted thoracoscopic surgery (B). Postoperative chest CT (C) showed no visible recurrence of cystic lesions or complications.

6 months after surgery showed no visible recurrence of cystic lesions or complications (Figure 2C).

\section{Discussion}

Bronchogenic cysts are benign congenital mediastinum masses for which the natural course are unknown (2). Because most bronchogenic cysts are thought to be asymptomatic, the best therapeutic strategies and optimal timing for surgery remain controversial $(3,4)$. However, because bronchogenic cysts can result in infection ,further complications due to compression of adjacent organs or malignant transformation, symptomatic and complicated bronchogenic cysts are usually surgically removed $(3,4)$. Because surgical excision can be hazardous and incomplete due to dense adhesion to surrounding vital organs after severe inflammation, drainage of complicated bronchogenic acute-phase cysts using bronchoscopy, mediastinoscopy, or percutaneous CT-guided methods has been performed to decompress them and relieve inflammation $(2,5)$. However, drainage is not considered a definitive treatment modality for complicated cysts because of recurrence or incomplete removal (6).

In our case, the patient's clinical history, symptoms, and signs suggested that he had deep neck infection and mediastinitis, which were confirmed by chest CT. Deep neck infection, mediastinitis, and the infected cysts were found after vomiting. The chronological order of events (drinking, vomiting, deep neck infection, mediastinitis, and the infected cyst) suggested that the most possible cause for the infected cyst is mediastinitis. We determined that the infected cyst was caused by acute mediastinitis due to esophageal injury, which is a rare cause of complicated bronchogenic cyst. An esophagogram was performed to investigate esophageal injury and showed no abnormalities. Therefore, we assumed that his vomiting episode 
induced esophageal micro-injury that was undetected by esophagogram, resulting in acute mediastinitis.

Severe adhesion to vital structures can be a clinically unfavorable condition for thoracoscopic surgery. To facilitate surgical dissection of the cystic lesion from severe adhesion, intraoperative fluid drainage was performed. We believe that cautious dissection of the cystic lesion after intraoperative drainage is useful in this setting. Intraoperative cyst drainage may help in the management of the lesion and preventing incomplete resection. In addition, when it has close relation to the esophagus and the airway, we should investigate possible communication with the esophagus and airway, preoperatively (1).

Because the VATS technique result in less damage to patients, even complicated bronchogenic cysts can be safely managed using the VATS technique $(3,6)$. To the best of our knowledge, this is the first case of infected bronchogenic cyst caused by mediastinitis and we describe how a surgical approach using VATS should be considered for treatment, even among patients with complicated bronchogenic cysts.

\section{Acknowledgements}

None.

\section{Footnote}

Conflicts of Interest: The authors have no conflicts of interest to declare.

Informed Consent: Written informed consent was obtained from the patient for publication of this manuscript and any accompanying images.

\section{References}

1. Lee DH, Park CK, Kum DY, et al. Clinical characteristics and management of intrathoracic bronchogenic cysts: a single center experience. Korean J Thorac Cardiovasc Surg 2011;44:279-84.

2. Weber T, Roth TC, Beshay M, et al. Video-assisted thoracoscopic surgery of mediastinal bronchogenic cysts in adults: a single-center experience. Ann Thorac Surg 2004;78:987-91.

3. De Giacomo T, Diso D, Anile M, et al. Thoracoscopic resection of mediastinal bronchogenic cysts in adults. Eur J Cardiothorac Surg 2009;36:357-9.

4. Kirmani B, Kirmani B, Sogliani F. Should asymptomatic bronchogenic cysts in adults be treated conservatively or with surgery? Interact Cardiovasc Thorac Surg 2010;11:649-59.

5. Hernández-Solís A, Cruz-Ortiz H, Gutiérrez-Díaz Ceballos $\mathrm{ME}$, et al. Bronchogenic cysts. Importance of infection in adults. Study of 12 cases. Cir Cir 2015;83:112-6.

6. Guo C, Mei J, Liu C, et al. Video-assisted thoracic surgery compared with posterolateral thoracotomy for mediastinal bronchogenic cysts in adult patients. J Thorac Dis 2016;8:2504-11.
Cite this article as: Kim YH, Kim JJ, Choi SY, Jeong SC, Kim IS. Complete thoracoscopic excision of an infected bronchogenic cyst due to mediastinitis. J Thorac Dis 2017;9(11):E979-E981. doi: 10.21037/jtd.2017.08.172 